\title{
High Pressure FAST of Nanocrystalline Barium Titanate
}

\author{
Martin B. Fraga a , Jean-Pierre Delplanque a , Nancy Yang b, \\ Enrique J. Lavernia ${ }^{\mathrm{d}}$, Todd C. Monson ${ }^{\mathrm{e}}$ \\ ${ }^{a}$ University of California Davis, Davis, California, USA \\ ${ }^{\mathrm{b}}$ Sandia National Laboratories, Livermore, California, USA \\ ${ }^{\mathrm{c}}$ University of California Irvine, Irvine, USA \\ d Sandia National Laboratories, Albuquerque, USA
}

\author{
Corresponding Author \\ name: Martin B. Fraga \\ e-mail: mbfraga@ucdavis.edu \\ tel: +1-(530)-752-9568 \\ address: 3001 Ghausi Hall, 1 Shields Ave, Davis, CA 95616
}

\begin{abstract}
This work studies the microstructural evolution of nanocrystalline $(<1 \mu \mathrm{m})$ barium titanate $\left(\mathrm{BaTiO}_{3}\right)$, and presents high pressure in field-assisted sintering (FAST) as a robust methodology to obtain $>100 \mathrm{~nm} \mathrm{BaTiO}_{3}$ compacts. Using FAST, two commercial $\sim 50 \mathrm{~nm}$ powders were consolidated into compacts of varying densities and grain sizes.

Microstructural inhomogeneities were investigated for each case, and an interpretation is developed using a modified Monte Carlo Potts (MCP) simulation. Two recurrent microstructural inhomogeneities are highlighted, heterogeneous grain growth and lowdensity regions, both ubiqutously present in all samples to varying degrees. In the worst cases, HGG presents an area coverage of $52 \%$. Because HGG is sporadic but homogenous throughout a sample, the catalyst (e.g., the local segregation of species) must be, correspondingly, distributed in a homogenous manner. MCP demonstrates that in such a case, a large distance between nucleating abnormal grains is required —otherwise abnormal grains prematurely impinge on each other, and their size is not distinguishable from that of normal grains. Compacts sintered with a pressure of $300 \mathrm{Mpa}$ and temperatures of $900{ }^{\circ} \mathrm{C}$, were $99.5 \%$ dense and had a grain size of $90 \pm 24 \mathrm{~nm}$. These are unprecedented results for commerical $\mathrm{BaTiO}_{3}$ powders or any starting powder of $50 \mathrm{~nm}$ particle size-other authors have used $16 \mathrm{~nm}$ lab-produced powder to obtain similar results.
\end{abstract}

Keywords: barium titanate (D); sintering (A); grain size (B); spark plasma sintering 


\section{Introduction}

Barium titanate (BaTiO3) is a ferroelectric compound whose properties render it well suited for its application in multi-layered ceramic capacitors (MLCC) [1] and other microelectronic devices [2]. This ceramic has a perovskite crystal structure, which assumes a tetragonal symmetry at room temperature [3]. $\mathrm{BaTiO}_{3}$ then undergoes a tetragonal-tocubic phase transition when temperatures exceed the Curie (transition) point. Subsequent phase transitions are well documented at lower temperatures [4,5]. While these phenomena have evoked multiple interpretations [6-8], the resulting ferroelectric and dielectric properties have maintained this material's industrial relevance.

However, the ever-increasing demand for smaller electronic components and more efficient designs require a reduction in the grain size of any ceramics used therein. In recent years, this trend has led to a need for nanocrystalline ceramics, which introduces a number of new challenges. The first challenge, which has largely been solved, is the consolidation of nanocrystalline $\mathrm{BaTiO}_{3}$ powders. Various processing approaches have been studied, such as hot isostatic pressing [9,10], conventional sintering [11,12], two-step sintering [13], and field-assisted sintering techniques (FAST) [13-18]. All these routes affirm powder sintering as a straightforward approach to generate fully dense materials from ceramic powders.

Other challenges are intrinsic to nanocrystalline $\mathrm{BaTiO}_{3}$ ceramics. The first arises from the high volume fraction of grain boundary atoms, which has repercussions in ionic materials_-as described by Joachim Maier [19]—leading to changes in the local chemistry and electric behavior. In addition, these paraelectric grain boundaries have a diluting effect on the dielectric behavior. Finally, the similar size between the equilibrium ferroelectric 
domains and the grains fixes the domain wall density (which contributes to the dielectric properties), and induces strains into the lattice. This strain is relaxed through a lattice distortion from the tetragonal symmetry, to an observed pseudo-cubic symmetry [20,21]. In nanocrystalline $\mathrm{BaTiO}_{3}$ materials the combined effects of the aforementioned phenomena lead to a negative trend of decreasing dielectric response with decreasing grain size [21-23]. In order to elucidate the onset and repercussions of these phenomena, the homogeneity of sintered samples is paramount.

In an effort to limit grain growth and thereby obtain a homogeneous microstructure, research in (FAST) has been prompted by the objective of decreasing the sintering times and temperatures required for full consolidation. This objective can be achieved by, for example, superimposing a thermal field with a pulsed current and a uniaxial pressure. FAST has emerged as a viable alternative to traditional sintering methods for a wide range of materials including, metals (TiAl alloys) [24], ceramics (B4C [25] and BaTiO3 [13-18]) and composites (carbon-fiber reinforced $\mathrm{Al}$ [26] and carbon nanotube reinforced $\mathrm{Ni}$ [27]). FAST offers several advantages relative to conventional sintering and other consolidation techniques. These advantages include: [28-31] (a) high thermal efficiency due to joule heating directly transforming an electric field into thermal energy; (b) rapid heating rates, also enabled by joule heating from a high pulse power source (up to $5000 \mathrm{~A}$ ), which allow scientists to rapidly reach the temperatures needed for densification (minimizing surface diffusion); and (c) the uniaxial pressure which enables the efficient packing of particles and leads to denser samples. Several other effects of the electric field have been observed in specific materials, which largely depend on their intrinsic electrical and thermal properties [29]. As shown in Table 1, various research groups around the world have been quite 
successful using this technique to produce dense, nanocrystalline BaTiO3 samples. Takeuchi et al. reported one of the first FAST-sintered BaTiO3 samples with a grain size smaller than $600 \mathrm{~nm}$ [14]. By using powder with high surface areas and high pressures, researchers such as Deng et al. produced full density samples at lower temperatures [20]. In turn, they achieved minimal grain growth and higher densities by increasing the heating rate and lowering the starting grain size and sintering temperature.[12] Subsequently, several research groups utilized FAST to produce a variety of nanocrystalline samples with high density (>95 \%). Despite the positive results, most of published studies do not describe microstructures in detail. Furthermore, some of the best results found in the literature are undermined by inconsistencies in their reporting. One example is found in the 2009 publication by Deng et al. 2009, which reported grain sizes of 20nm in compacts $99 \%$ dense. Not only was statistical information (such as standard deviations or histograms) not provided, but-more importantly — the scanning electron microscope (SEM) micrograph presented in their publication shows an average grain size well above 20nm (This author estimates a 40-50 $\mathrm{nm}$ average using ImageJ). In addition, the publication in question only reports relative densities; no actual density values are reported, and no mention of how the theoretical densities are estimated. This second issue is also seen in the work by Curecheriu et al. 2012, where they claim $90 \mathrm{~nm}$ and $99 \%$ density. Relative density is not meaningful when a theoretical value is not discussed, particularly in $\mathrm{BaTiO}_{3}$, where (as some of these same authors explain) there is a strong connection between grain size and lattice parameter. A final, yet fundamental, omission is any characterization of the homogeneity of the microstructure within the samples produced.

In fact, irregularities such as abnormal grain growth (AGG), which are commonly 
found in sintered $\mathrm{BaTiO}_{3}$ systems [32-34], are not mentioned the aforementioned publications. This phenomenon, which broadly refers to the exaggerated growth of certain grains at the expense of the surrounding normal grains (NGs), leads to a wide grain size distribution in the final microstructure. In this work, heterogeneous grain growth (HGG) is the term used to described the observed growth which yields regions of small grains (matrix of normal grains) and discontinuously large grains (abnormal grains). While similar to AGG in definition, the onset of HGG is sufficiently different that a distinction needs to be made to avoid confusion. On the other hand, the driving forces hypothesized are similar as will be described in subsequent sections. An additional concern with the literature is that none of the aforementioned investigations compare different commercial powders with similar specifications, rendering it difficult to draw conclusions as to the precise influence of starting powder characteristics and the properties of fabricated materials.

In view of the above discussion, the objectives of this study are threefold. First, to demonstrate the pervasiveness of irregularities in the microstructure of FAST-sintered BaTiO3 ceramics. Second, to provide insight into the mechanisms that govern the formation of such irregularities. Third, to propose processing strategies that can be implemented to mitigate them, while resulting in fully-dense samples and minimal grain growth. To satisfy these goals, a series of experiments were completed to observe the microstructural evolution of this material under different, changing parameters. The first set of experiments consisted of sintering four samples at different temperatures— $850{ }^{\circ} \mathrm{C}$, $900{ }^{\circ} \mathrm{C}, 950^{\circ} \mathrm{C}$ and $1000{ }^{\circ} \mathrm{C}$ - to determine the ideal temperature to minimize grain growth, while maximizing density and microstructural homogeneity. The samples were processed into compacts $2.5 \mathrm{~mm}$ in height and $19 \mathrm{~mm}$ in diameter. The heating rate was set to 
$100{ }^{\circ} \mathrm{C} / \mathrm{min}$, and the uniaxial pressure to $100 \mathrm{MPa}$. After sintering, all samples were annealed at $800^{\circ} \mathrm{C}$ for five hours and characterized. In the second set of experiments, a $900{ }^{\circ} \mathrm{C}$ heating profile was used to examine two sintering pressures; $100 \mathrm{MPa}$ and $300 \mathrm{MPa}$. In order to achieve these pressures, a two-stage $9 \mathrm{~mm}$ mold (also referred to as "die” in other publications) was utilized. Samples with a $1 \mathrm{~mm}$ height were sintered and characterized. Finally, a second commercial $\mathrm{BaTiO}_{3}$ powder from a different manufacturer was compared at $300 \mathrm{MPa}$ in order to gauge how much the starting powder can affect the final microstructure. This final route is presented as an equal or superior alternative methodology to that presented in the work from previous authors [13,17].

In parallel with the experiments described above, Monte Carlo Potts (MCP) simulations were used in an effort to provide fundamental insight into the microstructural evolution of heterogeneous growth (HGG) in this material. MCP captures the interaction of different states in a lattice through the application of statistical methods that predict the most probable outcome for a particular event [35-37]. Using methods described in detail in other publications [36,37], curvature-driven grain growth can be simulated by assessing the probability of a change in orientation at a selected site based on the resulting energy change. Normal grain growth has been modeled in two and three dimensions [38,39]. The success of this technique (and its application in this paper) lies in its ability of resolving meso-scale problems with reasonable computational complexity and effort. However, there are two main caveats hindering MCP. The first problem lies in the indeterminate nature of sites, and the inability of resolving information below their length-scale; such as local concentration inhomogeneities. The second problem is the lack of an energetic and temporal scale, which makes the calculation of certain relevant parameters (such as grain boundary mobility) very 
challenging. This issue is particularly limiting for sintering, where frequently the resolve is to connect the microstructure evolution with respect to time. However, the goal of the simulations as applied in this paper is to assess hypotheses stemming from the experimental observations rather than providing quantitative predictions.

\section{Methods}

The present work studied two types of commercially available $\mathrm{BaTiO}_{3}$ powder.

Table 2 shows the properties of both, as claimed by the respective manufacturers. In addition, both manufacturers claim that the powders are clustered into soft agglomerates. The first one was purchased from TPL, Inc. (Albuquerque, New Mexico). The second powder was obtained from Sakai Chemical Industry co., Itd. (Osaka, Japan). The particle size and $\mathrm{Ba} / \mathrm{Ti}$ ratio of these powders were corroborated with SEM and inductively coupled plasma mass spectrometry (ICPMS) respectively.

Field assisted sintering (FAST) was carried out in a Sumitomo SPS-825S system. Graphite molds were packed with the $\mathrm{BaTiO}_{3}$ powder in a nitrogen glovebox, and graphite foils used as interface between the powder and the plungers. The DC pulse pattern was set to twelve 3ms electric pulses and 8 ms of no current [28], and the vacuum held at $2 \mathrm{~Pa}$. A three-step heating profile was used, starting with a ten minute ramp up to the target temperature, which resulted in heating rates between $85-95^{\circ} \mathrm{C} / \mathrm{min}$. During this stepspecifically at $350^{\circ} \mathrm{C}$-a pressure of $100 \mathrm{MPa}$ (for the first experiments) was applied. This pressure was used to prevent the fracture of the graphite mold [40], and because it had previously led other authors to favorable results [17,41]. The second step consisted of holding the temperature and the pressure constant for six minutes. At the end of this step, 
pressure was released immediately. In the final step, the mold was left to cool in vacuum through the contact with the water-cooled arms of the FAST system.

An annealing step is required after sintering in order to restore the stoichiometry of the resulting compacts, which are reduced by the low-oxygen atmosphere in the FAST system. In order to determine the adequate annealing, samples were annealed at $800{ }^{\circ} \mathrm{C}$ for 4, 8 and 12 hours, and characterized. XRD and SEM grain size analysis were utilized to ascertain good stoichiometry and minimal grain growth during annealing.

Density measurements were obtained by the Archimedes method using a Mettler Toledo XS104 balance. X-ray Diffraction (XRD) spectra were collected on a Scintag XDS2000 powder diffractometer equipped with a graphite monochromator using $\mathrm{Cu} \mathrm{Ka}$ radiation. Raman spectroscopy analysis was performed using a Reinshaw System 1000 Laser Raman Microscope with a Spectra-Physics Ar-ion 514.5nm laser. Samples for XRD and Raman were ground in order to remove any surfaces affected by the annealing, and polished down to $3 \mu \mathrm{m}$ (diamond suspension). All the microstructure investigation was conducted on a FEI 430 NanoSEM system under a working voltage of 5-10kV and a spot size of 2-3. Grain analysis data was gathered from SEM micrographs of fractured surface, using a minimum of 200 grains.

Monte Carlo Potts simulations were used to provide insight into some of the conclusions drawn from experimental evidence. These simulations were performed with the SPPARKS simulation package [42] on a System76 computer equipped with an Intel i73930K CPU clocked at 3.20GHz. Due to the need for large abnormal grains, two dimensional lattices were used with 2500x2500 lattice units (LUs). The pinned growth algorithm was adapted from the module included in the SPPARKS libraries with a few 
minor modifications. The corresponding algorithm and Hamiltonian are outlined in the dissertation by Guebels et al [37]. Following their work, a simulation temperature of 1.0 was selected to prevent lattice faceting from lower temperatures, and to prevent artificial unpinning at higher temperatures. In order to maintain a consistent starting point, all cases were initialized by growing the matrix to a nominal average grain size of 20 units (using the exact parameters for all runs). Pins were then laid out on the matrix at the grain boundaries. A pin volume fraction of $7.5 \%$ was selected because, in preliminary runs, it resulted in a high density of grains per area, while maintaining a grain size large enough to overcome artifacts from temperature fluctuations. Abnormal grain nuclei (described in detail in the discussion), AGN, were dispersed on the matrix in a hexagonal distribution with the desired average distance. In order to generate AGNs, a new parameter (kinetic state, or k-state) was added to each site. This k-state parameter relates to the boundary kinetics of the grain and can take two values, 0 (normal grain) or 1 (abnormal grain). When a spin flip event occurs, the code checks the k-state, and if the grain is abnormal the temperature is increased by 0.1 . Upon a successful flip, the target site takes the orientation (as with classical MCP) and the k-state of the origin site. Preliminary results determined this value to be sufficient for the unpinning of the abnormal grains from the matrix, without overwhelming the stochastic nature of these events. Once the AGNs were laid out, the simulation was left to run until a fully-pinned microstructure, dominated by abnormal grains, was achieved. Due to the nature of MCP, grain growth will occur indefinitely if pinning can be overcome. It is therefore important to determine a reasonable number of Monte Carlo steps (MCS). The criterion used was that the doubling the total number of MCS should lead to an average growth in abnormal grains of less than 10\%. Running 300,000 MCS demonstrated that, for 
all random seeds, the criterion was met at 150,000 MCS. The reproducibility of all results was ascertained by running three simulations of the same case with a different random seed value.

\section{Results}

In the first set of experiments, desirable densities ( $>95 \%$ ) were achieved at temperatures above $900{ }^{\circ} \mathrm{C}$. The nominal grain size average increased with sintering temperature, as shown in Table 3. There is a considerable step between the standard deviations of samples below and above $900{ }^{\circ} \mathrm{C}$ due to increasing inhomogeneous growth of grains. In addition, the color of the as-sintered samples corresponds with their density; low density ( $<90 \%)$ samples are white, and high density (>90 \%) samples are increasingly dark blue. This blue color is due to the reduction of BaTiO3 in the FAST environment.

The XRD patterns of FAST-sintered $\mathrm{BaTiO}_{3}$ samples in Figure 1 show all the peaks corresponding to cubic $\mathrm{BaTiO}_{3}$, with some barium carbonate impurity peaks. Mild peak splitting is observed in some of the $\{200\}$ peaks, but the broadening caused by the small grain size and the strain make deconvolution difficult. From the observable splitting, samples above $900{ }^{\circ} \mathrm{C}$ present tetragonal symmetry. On the other hand, the evident lack of splitting in all peaks below this sintering temperature implies cubic symmetry [41,43].

Figure 2 presents the Raman spectra for samples sintered at the four target temperatures after being annealed for $5 \mathrm{hrs}$ at $800{ }^{\circ} \mathrm{C}$. All samples present first-order vibrational peaks at $252 \mathrm{~cm}^{-1}, 306 \mathrm{~cm}^{-1}, 515 \mathrm{~cm}^{-1}$ and $715 \mathrm{~cm}^{-1}$. These peaks correspond to the Raman events A1(TO), B1;E(TO-LO), E(TO);A1(TO), and E(LO);A1(LO) respectively. The events at $306 \mathrm{~cm}^{-1}$ and $715 \mathrm{~cm}^{-1}$ are associat ed with tetragonal symmetries, and should 
become suppressed after a tetragonal-to-cubic transition [44,45]. According to Raman, all samples present tetragonal character. These crystallographic results from Raman and XRD agree with observations made by previous authors [22,41, 44,46].

Figure 3 includes three SEM micrographs which identify the three distinct regions observed in all samples; the matrix, abnormal regions, and porous regions. The matrix, (a), is comprised of ultrafine grains and underwent full densification. Within this normal matrix, sporadic subsets of grains grow into abnormally large grains; ranging from a few, to tens of microns (b). These formations caused by HGG did not present any evident patterns that could be linked to the initial powder. On the other hand, the third type of region (c in Figure 4) was composed of grains finer than the matrix, and consistently had ellipsoidal shapes. These regions were highly porous, with a notable lack of densification.

The first occurrence of HGG was observed at $900{ }^{\circ} \mathrm{C}$, covering approximately $8 \%$ of the area fraction. Small “islands' of these abnormal grains were randomly distributed throughout the matrix, as illustrated in Figure 4. With increasing temperatures, these islands coalesced to form networks of abnormal regions, covering $48 \%$ at $950{ }^{\circ} \mathrm{C}$ and $52 \%$ at $1000^{\circ} \mathrm{C}$.

The second set of experiments addressed the effect of pressure on the resulting compact, and whether the qualities of the powder might play a role; the results are outlined in Table 4. Only the maximum density values included for the low-pressure samples because there was significant variability in their density, as seen in Table 3. This variability was also observed in the smaller sample sintered with the same parameters. On the other hand, higher pressures eliminated this variability. This consideration is sufficient for the 
purpose of this paper, which is not meant to systematically assess the effects of pressure.

The small sizes of the grains in the high pressure samples hinder a good resolution under the SEM, making them estimates. As such, corresponding micrographs are presented in Figure 5 for the reader's benefit. This figure also includes micrograph c), which shows an instance of heterogenous grain size in the compacts prepared with Sakai powder. This region is almost ellipsoidal, around $11 \mu \mathrm{m}$ in width and $21 \mu \mathrm{m}$ in height, an insignificant fraction of what was observed in low-pressure samples.

\section{Discussion}

\subsection{Grain Size, Density and Crystallography}

This section addresses issues related to the grain size and density of sintered compacts. As expected from classical sintering theories, lower sintering temperatures yield samples with smaller grains and reduced densities. The pronounced density variability in samples sintered at $900{ }^{\circ} \mathrm{C}$ highlights this as a critical temperature in the densification process. Li et al., in their work with FAST-sintering of $\mathrm{BaTiO}_{3}$, also observed a pronounced sintering trend around this temperature with density leveling off above $900{ }^{\circ} \mathrm{C}$ [46]. In addition, the microstructure of the samples sintered at temperatures below $850-900{ }^{\circ} \mathrm{C}$ indicate that, with all other parameters unchanged, high density ( $>95 \%)$ is only achievable with a minimum grain size above $450 \mathrm{~nm}$. As such, using these parameters, a maximum sintering temperature between $900{ }^{\circ} \mathrm{C}$ and $950{ }^{\circ} \mathrm{C}$ optimize density and grain size.

The abrupt color and density differences between the $850^{\circ} \mathrm{C}$ and $900{ }^{\circ} \mathrm{C}$, and the $950{ }^{\circ} \mathrm{C}$ and $1000^{\circ} \mathrm{C}$ samples seem to indicate an abrupt transformation. The increased 
propensity to spontaneous fracturing observed in samples sintered above $950{ }^{\circ} \mathrm{C}$ lends some evidence to the transition. This fracturing occurred sporadically in certain samples, after sintering and before annealing. Minimal pressure was required, and it occurred only along certain planes (presumably weak interfaces).

In addition, The XRD and Raman results are in good agreement with the results of previous authors, who observe a gradual change from a tetragonal symmetry to pseudocubic symmetry. It is hypothesized here that the strains produced by this change could contribute to the onset of HGG that will be discussed in the next section.

\subsection{Heterogeneous Grain Growth and Agglomerate Effects}

This section discusses the inhomogeneities that marred the microstructure of the samples, and could affect their electrical properties. In every sample that reached a density higher that $90 \%$, HGG was observed to varying degrees. Temperature and pressure were not sufficient to completely eliminate this phenomenon. The evident pervasiveness of HGG in these FAST-sintered $\mathrm{BaTiO}_{3}$ samples requires further examination. At a first glance agglomeration in the powder might be a prime suspect for this phenomenon. As mentioned in the methods section, both manufacturers claim soft agglomerates. To corroborate this, both powders were dispersed with a sonication wand and observed under the SEM. A fisher sonic dismembrator model 300 was used set at 50 \% intensity. Each powder was placed into a small eppendorf tube and sonicated four times for thirty seconds. As seen in Figure 6 the TPL powder was only partially dispersed, while the Sakai powder dispersed completely. This positive co-relation indicates that harder agglomerates may play a role, but the Sakai powder still presented HGG; therefore, the degree to which 
agglomerates might have an effect is not clear.

The growth of HGG regions in the TPL powder suggests that agglomerates might not play the major role. If agglomerates were the cause, HGG would occur in agglomerates that meet certain conditions, and that abnormal regions would expand to the rest of the matrix. However, experimentally the expansion of HGG regions, seen in Figure 5, occurs not through the overwhelming growth of few grains, but rather by the activation of HGG in adjacent grains within an area. Agglomerate-induced abnormal growth was also eliminated as a possibility in their samples by authors working in Titania (TiO2) [39]. One possible process examined by several authors have linked AGG in BaTiO3 to faceted grain boundaries $[32,47,48]$. They claim that faceted boundaries do not act as perfect sinks or sources of atoms and vacancies, but rather create a barrier that hinder grain growth and densification mechanisms below a critical energy [48]. On the other hand, Baurer et al. reported contrary evidence, with matrix grains adjacent to abnormally-grown grains growing with no apparent effect from such potentials [34]. They connect AGG with solute drag effects in Ti-rich materials as generally described in the phase-field simulations by Kim and Park [49]. This could be the driving force for HGG.

The possibility that sites of solute segregation or secondary particles are the cause of the observed HGG was investigated with Monte Carlo Potts simulations. Using well established particle pinning methods [37,39,50], the first step was to achieve a complete pinning of the microstructure. Increasing the volume fraction of pins will decrease the equilibrium grain size of the lattice, which would allow more grains per simulation. On the other hand, too many pins will increase the time needed for grain unpinning and may deviate too far from the pinning expected to occur in such a ceramic. In these simulations, 
pins are meant to represent a drag force to grain growth and therefore their volume fraction does not necessarily need to correspond to that of impurities observed experimentally. After deciding on a volume fraction based on the work by Guebels et al. [37], HGG was forced on certain grains by modifying their local simulation temperatures. Higher simulation temperatures allows for higher fluctuations that enable the unpinning of boundaries. These initial, selected grains are henceforth defined "abnormal growth nuclei” (AGN). This forced HGG is artificial, but suffices to show how abnormal grains may interact and lead to the experimental observations. For this purpose, AGNs were laid out throughout the lattice in a hexagonal array). The distance between nuclei can be controlled easily by selecting the hexagon's edge length; this hexagonal dispersion of AGNs can be seen on Figure 7 (a and c) for two cases with different inter-AGN distances. Subsequent growth leads to an equilibrium grain size of the abnormal grains, which depends on the average distance between AGNs. The final growth for 150,000 MCS is shown in Figure 7 for cases with a nuclei distance of 50 (b) and 225 (d). The effect of nuclei distance (ranging from 50 to 225 lattice units) on the growth behavior of abnormal grains was compared to the experimental observations. The deviation in the size of grains grows considerably (and linearly) with increasing nuclei distances. Experimentally, regions with HGG tend to have relatively uniform grain size distributions. This implies that there is a uniform distribution in the grains that have the conditions to grow abnormally. On the other hand, to reach the observed size ratio between normal and abnormal grains, large AGN distances are required. As expected, the growth of individual AGNs was found to depend on the local environment. As seen on Table 5, all AGN distances used resulted in at least a hundred AGNs. Some AGs disappeared due to extremely constrained environments, or at the 
expense of other AGs. The average radius and standard deviation grow linearly with AGN distance, as Figure 9 also shows. To corroborate that AGs strongly impinge on each other, a calculated radius was defined by dividing the area by the final number of AGs. A good corelation was found with the actual measured values. As an additional measurement, growth constants were fitted to a power-law growth equation $\mathrm{D}=\mathrm{k}(\mathrm{MCS})^{\mathrm{n}}$. These values are only meant to show the general trends of growth, not physical properties. At small AGN distances, the AGs quickly meet and impinge on each other, leading to a sharp growth (large k) with a quick leveling off (low n). With increasing distance, the AGs have more freedom to grow uninhibited, which lead smaller $\mathrm{k}$ values and more reasonable $\mathrm{n}$ values (closer to those seen in normal growth (0.5).

The results reveal good self-consistency, with the standard deviations showing insignificant data overlap. With the assumption that only certain grains will meet the requirements for HGG, the simulations show that the experimental observations could be attributed to the proposed mechanism. Heavy pinning of the microstructure will restrict grain growth, a situation that could explain the limited growth of matrix grains between $900{ }^{\circ} \mathrm{C}$ and $950^{\circ} \mathrm{C}$. Assuming that the conditions for abnormal kinetics are not uncommon (an assumption supported by the experimental results), then the microstructure must have sporadic regions that meet the requirements for the activation of HGG. As shown in the simulations, if the distance between these sites is small, then the abnormal growth will occur rapidly, and the AGs will impinge on each other early on. In this case, the standard deviation is small and the grain size may not be noticeably larger than the matrix. In fact, this situation could be impossible to recognize with ex-situ observations. With increasing distance between AGNs, abnormal grains have increasing room and time to grow (as shown 
by the growth coefficients) as long as conditions are favorable. This leads to larger standard deviations. In addition, this case allows for large AG/NG ratios to occur, leading to noticeable differences between regions of normal and abnormal growth. Such an abrupt change in the activation of HGG could account for the similar abrupt jump in grain size observed in the $1000{ }^{\circ} \mathrm{C}$ samples. The experimental case at $950{ }^{\circ} \mathrm{C}$ shown in Figure 4 has an AG/NG ratio around 4, which is close to that found in the simulation case with an AGN distance of 225 lattice units (which has a ratio of 3.4). However, the high variability in grain size is not observed to the same degree experimentally. Such a discrepancy can be ascribed to the simplicity of the proposed model, but it may also simply be due to an overpinning of the simulation matrix. Regardless, these results require homogeneous distributions of solute segregation (or some other pin-inducing lattice artifact) within regions of HGG. AGG is usually described as a rare and stochastic process, but the experimental results show that, in contrast, HGG can be consistently obtained. While undeniably stochastic, the pervasiveness of this phenomenon highlights the notion that simple rules are in play, which all are adequately described by MCP. Replacing the forced HGG seed with a more accurate interpretation could turn this tool into a viable method of predicting and preventing HGG (and possibly AGG). In fact, MCP used in combination with anisotropic models, as described in other studies [51,52], could lead to more accurate description of the growth that occurs in $\mathrm{BaTiO}_{3}$ materials.

\subsection{Porous regions}

Ellipsoidal, porous regions with grains smaller than those in the matrix are equally prevalent in all of the TPL-prepared samples. The ellipsoidal shape of these regions could 
correspond to a distorted cross-section of the spherical agglomerates. By using a sintering pressure of 300MPa (explained in the next section), these regions were densified successfully, but the grains were still considerably smaller than those in the matrix. This success could be attributed to the strain affecting the interfacial potentials as described in the previous section [29]. Compacts sintered from the Sakai powder show similar ellipsoidal regions, but with larger grains rather than porosity. This inconsistency between powders could be evidence that these regions can take either route, depending on their geometry and their chemical nature. In the Sakai material, these regions cover a smaller area fraction than in the TPL counterparts, providing further evidence for the weaker nature of the agglomerates in the Sakai powder.

\subsection{High Pressure Samples}

HGG, like AGG, requires an abrupt jump in driving force for the mobility of certain grain boundaries, and requires grain growth. As such decreasing sintering temperature, and increasing heating rate and pressure affect the boundary kinetics and minmize grain growth-therefore they also hinder HGG. Decreasing sintering temperature was clearly insufficient from our results. Full density was never achieved this way, and HGG was never fully mitigated. Preliminary results from increasing heating rate were not encouraging. Achieving stable heating of the die was problematic, and the resulting compacts were not significant improvement. In addition, the temperature controller does not allow for precise control over the heating rate. This discrepancy with the literature may be due to the starting powder, which is larger than those used by other groups $[17,44]$. As such, high pressure 
was the only parameter left. Using $300 \mathrm{MPa}$ yielded full density, and minimal grain growth allowed a grain size of $120 \mathrm{~nm}$ in the TPL powder and $90 \mathrm{~nm}$ in the Sakai powder. This difference is due to the initial particle size and to the purity of the powder - a factor that also explains the difference in the extent of HGG. On the other hand, the extent of HGG and porous regions was small enough to be unmeasurable using the same technique as for the previous compacts.

\section{Conclusions}

In conclusion, this study examined the microstructural evolution of inhomogeneities in FAST-sintered, BaTiO3 at varying temperatures, and mitigated their occurrence by using high pressures. HGG and low-density regions were ubiquitously found throughout the microstructures of our low pressure samples, the former reaching coverage of up to $52 \%$ in the $1000^{\circ} \mathrm{C}$ samples. While reducing the sintering temperature was successful in hindering HGG, no samples produced in this study were entirely devoid of such inhomogeneities. HGG is proposed to be caused by solute drag, and the modified MCP simulations show that a large distance between AGN is needed to achieve the expected normal-to-abnormal grain size ratio found experimentally. Smaller distances lead to early impinging between abnormal grains, which result in grains marginally larger than those expected from normal growth. In addition, large distances also lead to a large variability in the size of abnormal grains. These results provide a connection between the distribution of the source of HGG, and the final microstructural observations. Using a more realistic description of the difference in kinetics between normal and abnormal grains could result in better alignment with the experimental observations. Further work would be required to imbue this model 
with predictability qualities.

In trying to eliminate HGG and porous regions with high pressure, fully dense nanocrystalline compacts were produced. Using TPL powder, the compacts had a density of $90.0 \%$ and a grain size of $120 \pm 36 \mathrm{~nm}$. Using Sakai powder, the compacts had a density of $99.5 \%$ and a grain size of $90 \pm 24 \mathrm{~nm}$. These results are comparable to some of the best results in the literature, yet the starting powder is commercial and more than five times larger. In both cases, inhomogeneities are successfully hindered and mostly eliminated. In this regard as well, the Sakai powder fared much better, with minimal HGG regions and no observed porosity.

\section{Acknowledgements}

The authors gratefully acknowledge the support of Sandia National Laboratories. The authors also wish to thank Dr. Susan Heidger of the Air Force Research Laboratory/High Power Microwave Electromagnetic Microwave Division for additional support of this work. Sandia National Laboratories is a multi-program laboratory managed and operated by Sandia Corporation, a wholly owned subsidiary of the Lockheed Martin Corporation, for the US Department of Energy's National Nuclear Security Administration under contract no. DE-AC04-94AL85000.

\section{References}

[1] C. Pithan, D. Hennings, R. Waser, Progress in the synthesis of nanocrystalline

BaTiO3 powders for MLCC, Int. J. Appl. Ceram. Technol. 2 (2005) 1-14. 
[2] A. Gruverman, A. Kholkin, Nanoscale ferroelectrics: processing, characterization and future trends, Rep. Prog. Phys. 69 (2006) 2443-2474. doi:10.1088/0034-4885/69/8/R04.

[3] H. Megaw, Crystal structure of barium titanate, MACMILLAN MAGAZINES LTD PORTERS SOUTH, 4 CRINAN ST, LONDON N1 9XW, ENGLAND, 1945.

[4] A. von Hippel, Ferroelectricity, Domain Structure, and Phase Transitions of Barium Titanate, Rev. Mod. Phys. 22 (1950) 221-237. doi:10.1103/RevModPhys.22.221.

[5] V. Polinger, Ferroelectric phase transitions in cubic perovskites, J. Phys. Conf. Ser. 428 (2013) 12026. doi:10.1088/1742-6596/428/1/012026.

[6] Y. Chiang, Physical ceramics, J. Wiley, New York, 1997.

[7] Q. Zhang, T. Cagin, W.A. Goddard, The ferroelectric and cubic phases in BaTiO3 ferroelectrics are also antiferroelectric, Proc. Natl. Acad. Sci. 103 (2006) 14695-14700. doi:10.1073/pnas.0606612103.

[8] B. Zalar, V.V. Laguta, R. Blinc, NMR Evidence for the Coexistence of OrderDisorder and Displacive Components in Barium Titanate, Phys. Rev. Lett. 90 (2003) 37601. doi:10.1103/PhysRevLett.90.037601.

[9] Y. Hirata, A. Nitta, S. Sameshima, Y. Kamino, Dielectric properties of barium titanate prepared by hot isostatic pressing, Mater. Lett. 29 (1996) 229-234. doi:10.1016/S0167-577X(96)00149-8.

[10] H. Maiwa, Piezoelectric properties of BaTiO3 ceramics prepared by hot isostatic pressing, J. Ceram. Soc. Jpn. 121 (2013) 655-658.

[11] N. Halder, D. Chattopadhyay, A. Das Sharma, D. Saha, A. Sen, H.S. Maiti, Effect of sintering atmosphere on the dielectric properties of barium titanate based capacitors, Mater. Res. Bull. 36 (2001) 905-913. doi:10.1016/S0025-5408(01)00540-2. 
[12] K. Maeda, I. Fujii, K. Nakashima, G. Fujimoto, K. Suma, T. Sukigara, S. Wada, Preparation of barium titanate porous ceramics and their sensor properties, J. Ceram. Soc. Jpn. 121 (2013) 698-701.

[13] X. Deng, D. Li, J. Li, X. Wang, L. Li, Preparation of nanocrystalline BaTiO3 ceramics, Sci. China Ser. E Technol. Sci. 52 (2009) 1730-1734. doi:10.1007/s11431-0090033-4.

[14] T. Takeuchi, C. Capiglia, N. Balakrishnan, Y. Takeda, H. Kageyama, Preparation of fine-grained BaTiO3 ceramics by spark plasma sintering, J. Mater. Res. 17 (2001) 575-581. [15] Z. Valdez-Nava, S. Guillemet-Fritsch, C. Tenailleau, T. Lebey, B. Durand, J.Y. Chane-Ching, Colossal dielectric permittivity of BaTiO3-based nanocrystalline ceramics sintered by spark plasma sintering, J. Electroceramics. 22 (2009) 238-244. doi:10.1007/s10832-007-9396-8.

[16] S. Yoon, J. Dornseiffer, Y. Xiong, D. Grüner, Z. Shen, S. Iwaya, C. Pithan, R. Waser, Spark plasma sintering of nanocrystalline BaTiO3-powders: Consolidation behavior and dielectric characteristics, J. Eur. Ceram. Soc. 31 (2011) 1723-1731. doi:10.1016/j.jeurceramsoc.2011.03.035.

[17] L. Curecheriu, S.-B. Balmus, M.T. Buscaglia, V. Buscaglia, A. Ianculescu, L. Mitoseriu, Grain Size-Dependent Properties of Dense Nanocrystalline Barium Titanate Ceramics, J. Am. Ceram. Soc. 95 (2012) 3912-3921. doi:10.1111/j.15512916.2012.05409.x.

[18] A.A. Kholodkova, M.N. Danchevskaya, Y.D. Ivakin, G.P. Muravieva, Synthesis of fine-crystalline tetragonal barium titanate in low-density water fluid, J. Supercrit. Fluids. 105 (2015) 201-208. doi:10.1016/j.supflu.2015.05.004. 
[19] J. Maier, Thermodynamic aspects and morphology of nano-structured ion conductors: Aspects of nano-ionics Part I, Solid State Ion. 154-155 (2002) 291-301. doi:10.1016/S0167-2738(02)00499-X.

[20] X. Deng, X. Wang, H. Wen, L. Chen, L. Chen, L. Li, Ferroelectric properties of nanocrystalline barium titanate ceramics, Appl. Phys. Lett. 88 (2006) 252905. doi:10.1063/1.2213508.

[21] M.H. Frey, D.A. Payne, Grain-size effect on structure and phase transformations for barium titanate, Phys. Rev. B. 54 (1996) 3158-3168. doi:10.1103/PhysRevB.54.3158.

[22] G. Arlt, D. Hennings, G. de With, Dielectric properties of fine-grained barium titanate ceramics, J. Appl. Phys. 58 (1985) 1619-1625. doi:doi:10.1063/1.336051.

[23] T. Teranishi, T. Hoshina, T. Tsurumi, Wide range dielectric spectroscopy on perovskite dielectrics, Mater. Sci. Eng. B. 161 (2009) 55-60. doi:10.1016/j.mseb.2008.11.039.

[24] A. Couret, G. Molénat, J. Galy, M. Thomas, Microstructures and mechanical properties of TiAl alloys consolidated by spark plasma sintering, Intermetallics. 16 (2008) 1134-1141. doi:10.1016/j.intermet.2008.06.015.

[25] P. Badica, H. Borodianska, S. Xie, T. Zhao, D. Demirskyi, P. Li, A.I.Y. Tok, Y. Sakka, O. Vasylkiv, Toughness control of boron carbide obtained by spark plasma sintering in nitrogen atmosphere, Ceram. Int. 40 (2014) 3053-3061.

doi:10.1016/j.ceramint.2013.09.141.

[26] G. Lalet, H. Kurita, T. Miyazaki, A. Kawasaki, J.-F. Silvain, Microstructure of a carbon fiber-reinforced aluminum matrix composite fabricated by spark plasma sintering in 
various pulse conditions, J. Mater. Sci. 49 (2014) 3268-3275. doi:10.1007/s10853-0148032-7.

[27] J. Nguyen, T.B. Holland, H. Wen, M. Fraga, A. Mukherjee, E. Lavernia, Mechanical behavior of ultrafine-grained Ni-carbon nanotube composite, J. Mater. Sci. 49 (2014) 2070-2077. doi:10.1007/s10853-013-7897-1.

[28] W. Chen, U. Anselmi-Tamburini, J.E. Garay, J.R. Groza, Z.A. Munir, Fundamental investigations on the spark plasma sintering/synthesis process: I. Effect of dc pulsing on reactivity, Mater. Sci. Eng. A. 394 (2005) 132-138. doi:10.1016/j.msea.2004.11.020. [29] Z.A. Munir, U. Anselmi-Tamburini, M. Ohyanagi, The effect of electric field and pressure on the synthesis and consolidation of materials: A review of the spark plasma sintering method, J. Mater. Sci. 41 (2006) 763-777. doi:10.1007/s10853-006-6555-2. [30] R. Orrù, R. Licheri, A.M. Locci, A. Cincotti, G. Cao, Consolidation/synthesis of materials by electric current activated/assisted sintering, Mater. Sci. Eng. R Rep. 63 (2009) 127-287. doi:10.1016/j.mser.2008.09.003.

[31] F. Maglia, I.G. Tredici, U. Anselmi-Tamburini, Densification and properties of bulk nanocrystalline functional ceramics with grain size below 50 nm, J. Eur. Ceram. Soc. 33 (2013) 1045-1066. doi:10.1016/j.jeurceramsoc.2012.12.004.

[32] B.-K. Lee, S.-Y. Chung, S.-J.L. Kang, Grain boundary faceting and abnormal grain growth in BaTiO3, Acta Mater. 48 (2000) 1575-1580. doi:10.1016/S1359-6454(99)004346.

[33] S. Subramaniam, R.D. Roseman, Dynamic Observations of Abnormal Grain-growth Occurring in Barium Titanate During Sintering, Microsc. Microanal. 10 (2004) 1066-1067. doi:10.1017/S1431927604886331. 
[34] M. Bäurer, S.-J. Shih, M.P. Harmer, D. Cockayne, M.J. Hoffmann, Abnormal grain growth in undoped strontium and barium titanate, Acta Mater. 58 (2010) 290-300. doi:10.1016/j.actamat.2009.09.007.

[35] J. Ashkin, E. Teller, Statistics of Two-Dimensional Lattices with Four Components, Phys. Rev. 64 (1943) 178-184. doi:10.1103/PhysRev.64.178.

[36] M.. Anderson, D.. Srolovitz, G.. Grest, P.. Sahni, Computer simulation of grain growth-I. Kinetics, Acta Metall. 32 (1984) 783-791. doi:10.1016/0001-6160(84)90151-2. [37] C.A.P.N. Guebels, A Monte Carlo Potts Investigation of Microstructural Evolution: Particle Assisted Abnormal Grain Growth, 2011. http://adsabs.harvard.edu/abs/2011PhDT.......126G (accessed February 20, 2014). [38] Q. Yu, S.K. Esche, Three-dimensional grain growth modeling with a Monte Carlo algorithm, Mater. Lett. 57 (2003) 4622-4626. doi:10.1016/S0167-577X(03)00372-0.

[39] D.J. Srolovitz, M.P. Anderson, G.S. Grest, P.S. Sahni, Computer simulation of grain growth-III. Influence of a particle dispersion, Acta Metall. 32 (1984) 1429-1438. doi:10.1016/0001-6160(84)90089-0.

[40] R. Chaim, Densification mechanisms in spark plasma sintering of nanocrystalline ceramics, Mater. Sci. Eng. A. 443 (2007) 25-32. doi:10.1016/j.msea.2006.07.092. [41] V. Buscaglia, M.T. Buscaglia, M. Viviani, L. Mitoseriu, P. Nanni, V. Trefiletti, P. Piaggio, I. Gregora, T. Ostapchuk, J. Pokorný, J. Petzelt, Grain size and grain boundaryrelated effects on the properties of nanocrystalline barium titanate ceramics, J. Eur. Ceram. Soc. 26 (2006) 2889-2898. doi:10.1016/j.jeurceramsoc.2006.02.005.

[42] SPPARKS Users Manual, (n.d.). http://spparks.sandia.gov/doc/Manual.html (accessed July 27, 2014). 
[43] V.K. Pecharsky, P.Y. Zavalij, Fundamentals of powder diffraction and structural characterization of materials, Springer, New York, 2009. http://dx.doi.org/10.1007/978-0387-09579-0 (accessed November 12, 2014).

[44] X. Deng, X. Wang, H. Wen, A. Kang, Z. Gui, L. Li, Phase Transitions in Nanocrystalline Barium Titanate Ceramics Prepared by Spark Plasma Sintering, J. Am. Ceram. Soc. 89 (2006) 1059-1064. doi:10.1111/j.1551-2916.2005.00836.x.

[45] A.S. Chaves, F.C.S. Barreto, R.A. Nogueira, B. Zẽks, Thermodynamics of an eightsite order-disorder model for ferroelectrics, Phys. Rev. B. 13 (1976) 207-212. doi:10.1103/PhysRevB.13.207.

[46] B. Li, X. Wang, M. Cai, L. Hao, L. Li, Densification of uniformly small-grained BaTiO3 using spark-plasma-sintering, Mater. Chem. Phys. 82 (2003) 173-180. doi:10.1016/S0254-0584(03)00195-0.

[47] Y.-I. Jung, S.-Y. Choi, S.-J.L. Kang, Grain-Growth Behavior during Stepwise Sintering of Barium Titanate in Hydrogen Gas and Air, J. Am. Ceram. Soc. 86 (2003) $2228-2230$.

[48] S.-Y. Choi, S.-J.L. Kang, Sintering kinetics by structural transition at grain boundaries in barium titanate, Acta Mater. 52 (2004) 2937-2943. doi:10.1016/j.actamat.2004.02.039.

[49] S.G. Kim, Y.B. Park, Grain boundary segregation, solute drag and abnormal grain growth, Acta Mater. 56 (2008) 3739-3753. doi:10.1016/j.actamat.2008.04.007.

[50] E.A. Holm, M.A. Miodownik, A.D. Rollett, On abnormal subgrain growth and the origin of recrystallization nuclei, Acta Mater. 51 (2003) 2701-2716. doi:10.1016/S13596454(03)00079-X. 
[51] G.S. Grest, D.J. Srolovitz, M.P. Anderson, Computer simulation of grain growthIV. Anisotropic grain boundary energies, Acta Metall. 33 (1985) 509-520. doi:10.1016/0001-6160(85)90093-8.

[52] O.M. Ivasishin, S.V. Shevchenko, S.L. Semiatin, Implementation of exact grainboundary geometry into a 3-D Monte-Carlo (Potts) model for microstructure evolution, Acta Mater. 57 (2009) 2834-2844. doi:10.1016/j.actamat.2009.02.034. 


\section{Figure Captions}

Figure 1: XRD patterns of BaTiO3 compacts FAST-sintered at four temperatures, and annealed at $800^{\circ} \mathrm{C}$ for 5 hours. Indexed peak positions for cubic BaTiO3 at displayed at the top of the figure. The intensity range is from 0 to 1400 counts per second.

Figure 2: Raman spectra of FAST-sintered BaTiO3 compacts at four temperatures, and annealed at $800^{\circ} \mathrm{C}$ for 5 hours. Raman events for BaTiO3 systems are marked by dashed lines, and labeled at the top.

Figure 3: SEM micrographs of a fractured surface of a BaTiO3 sample, sintered at $950^{\circ} \mathrm{C}$, where (a) shows a region with the nominal grain size average, (b) a region of abnormally large grains, and (c) a porous region.

Figure 4: SEM micrographs of fractured surfaces of BaTiO3 samples at different temperatures. Matrix and abnormal regions separated with white demarcations and marked by arrows.

Figure 5: SEM micrograph of fractured surfaces of FAST-sintered compact produced from: a) TPL powder and b) Sakai powder. Micrograph c) shows the occurrence of HGG in Sakai compacts. These compacts were sintered at $900^{\circ} \mathrm{C}$ for $10 \mathrm{~min}$ under $300 \mathrm{MPa}$ of pressure. 
Figure 6: Micrographs of TPL and Sakai Powders. a) and b) correspond to the as-received and de-agglomerated TPL powders respectively, and c) and d) correspond to the Sakai asreceived and de-agglomrated powders respectively.

Figure 7: Cropped images of simulated microstructures. Normal grains are colored white, abnormal grains dark gray, and boundaries and pins black. Figures (a) and (b) show cases with hexagonally-distributed, 75 LUs apart, abnormal grain nuclei and their growth after 150,000 MCS respectively. Figures (c) and (d) correspond to the case where AGNs are distributed 225 LUs apart. 
Table(s)

Table 1: Reported results from previous authors working on FAST-sintered BaTiO3 [13],

[14], [16]-[18]. These include the initial powder size (PS), sintering temperature (T), sintering pressure (P), resulting grain size (GS), and relative density $(\rho)$.

\begin{tabular}{llllll} 
Author & $\mathbf{P S}$ & $\mathbf{T}$ & $\mathbf{P}$ & $\mathbf{G S}$ & $\mathbf{\rho}$ \\
& $\mathbf{( n m )}$ & $\left({ }^{\circ} \mathbf{C}\right)$ & $\mathbf{( M P a )}$ & $\mathbf{( n m )}$ & $\mathbf{( \% )}$ \\
\hline Takeuchi (2001) & 60 & 1100 & $30-50$ & $<500$ & 95 \\
\hline Deng (2009) & $10-30$ & $820-850$ & 70 & $15-100$ & $95-99$ \\
\hline Yoon (2011) & $10-15$ & $950-1050$ & 75 & $250-650$ & $95-99$ \\
\hline Curecheriu (2012) & 16 & $1000-$ & 100 & 92 & 99 \\
& & 1250 & &
\end{tabular}

$\begin{array}{llllll}\text { Kholodkova (2015) } & 37.6 & 1140 & 50 & 188 & --\end{array}$


Table 2: Manufacture details on the commercial powders used.

\begin{tabular}{lllll}
\hline Source & Specific Surface Area & Particle Size & Purity & Ba/Ti ratio \\
& $(\mathbf{m} 2 / \mathbf{g})$ & $(\mathbf{n m})$ & $\mathbf{( \% ~ B a T i O 3 )}$ & \\
\hline TPL & $15-18$ & 50 & 99.5 & $0.995-1.01$ \\
\hline Sakai & 22.2 & 47 & 99.98 & 1.00 \\
\hline
\end{tabular}


Table 3: BaTiO3 samples FAST-sintered at four different temperatures.

\begin{tabular}{|c|c|c|c|c|}
\hline Property & $850^{\circ} \mathrm{C}$ & $900^{\circ} \mathrm{C}$ & $950^{\circ} \mathrm{C}$ & $1000^{\circ} \mathrm{C}$ \\
\hline \# Samples & 3 & 4 & 3 & 3 \\
\hline Heating Rate $\left({ }^{\circ} \mathrm{C} / \mathrm{min}\right)$ & 97.5 & 92.5 & 87.5 & 82.5 \\
\hline Avg. Grain Size $(\mathrm{nm})^{[\mathrm{a}]}$ & $137 \pm 50$ & $306 \pm 96$ & $292 \pm 448$ & $462 \pm 618$ \\
\hline Density (g/cm3) & $4.77 \pm 0.2$ & $5.46 \pm 0.3^{[b]}$ & $5.79 \pm 0.02$ & $5.80 \pm 0.06$ \\
\hline Density (\%) & $79.2 \pm 3$ & $90.6 \pm 5^{[b]}$ & $96.09 \pm 0.3$ & $96.31 \pm 1$ \\
\hline As-sintered Color & white & white-blue & blue-green & dark blue-green \\
\hline \multicolumn{5}{|c|}{ [a] Average grain size is calculated over entire histogram. Due to abnormal grain growth, } \\
\hline \multicolumn{5}{|c|}{ average can be much larger than the mode) [b] Samples sintered near $900^{\circ} \mathrm{C}$ seem to } \\
\hline
\end{tabular}


Table 4: Samples FAST-sintered at $900^{\circ} \mathrm{C}$ with varying conditions. Resulting densities ( $\rho$ ) and average grain sizes (GS) are shown. For each sample, the maximum (not the average) density is presented.

\begin{tabular}{|c|c|c|c|}
\hline Sample & $\begin{array}{c}\rho \\
(\mathrm{g} / \mathrm{cm} 3)\end{array}$ & $\begin{array}{c}\text { Rel. } \rho \\
\text { (\%) }\end{array}$ & GS (nm) \\
\hline $900^{\circ} \mathrm{C}, \mathrm{TPL}, \varnothing 19 \times 3 \mathrm{~mm}, 100 \mathrm{MPa}$ & 5.72 & 95.0 & $306 \pm 96$ \\
\hline $900^{\circ} \mathrm{C}, \mathrm{TPL}, \varnothing 9 \times 1 \mathrm{~mm}, 100 \mathrm{MPa}$ & 5.80 & 96.4 & $260 \pm 57$ \\
\hline $900^{\circ} \mathrm{C}, \mathrm{TPL}, ø 9 \times 1 \mathrm{~mm}, 300 \mathrm{MPa}$ & 5.96 & 99.0 & $120 \pm 36$ \\
\hline $900^{\circ} \mathrm{C}$, Sakai ø9x1mm, 300MPa & 5.99 & 99.5 & $90 \pm 24$ \\
\hline
\end{tabular}


Table 5: Information on the growth of abnormal grains (AG) in a 2500x2500 lattice with a temperature of 1.0 and a local AGG temperature of 1.1. AGN distance corresponds to the average spacing between abnormal grain nuclei. In addition the table shows the starting and final (after 150,000 Monte Carlo steps) number of abnormal grains, the average and standard deviation of abnormal grain size, and a calculated radius assuming perfect tiling of spherical particles (i.e., volume/number of grains) The subsequent, $\mathrm{k}$ and $\mathrm{n}$, parameters are the growth constants determined by fitting the growth curves to the equation $\mathrm{D}=\mathrm{k}(\mathrm{MCS}) \mathrm{n}$.

\begin{tabular}{|c|c|c|c|c|c|c|c|}
\hline $\begin{array}{l}\text { AGN } \\
\text { distance }\end{array}$ & $\begin{array}{l}\text { Starting } \\
\text { \# of AGs }\end{array}$ & $\begin{array}{l}\text { Final \# } \\
\text { of AGs }\end{array}$ & $\begin{array}{r}\text { Radius } \\
\text { Average }\end{array}$ & $\begin{array}{l}\text { Radius } \\
\text { Std. Dev. }\end{array}$ & $\begin{array}{r}\text { Calculated } \\
\text { Radius }\end{array}$ & $\mathbf{k}$ & $\mathbf{n}$ \\
\hline 50 & 2850 & 1578 & 30.7 & 13 & 26.4 & 13.9 & 0.136 \\
\hline 75 & 1287 & 796 & 41.9 & 19.6 & 39.3 & 10.7 & 0.191 \\
\hline 150 & 301 & 184 & 85.7 & 44.5 & 81.3 & 4.46 & 0.310 \\
\hline 225 & 110 & 84 & 118 & 57.6 & 134.5 & 4.06 & 0.338 \\
\hline
\end{tabular}


Figure(s)

Figure 1

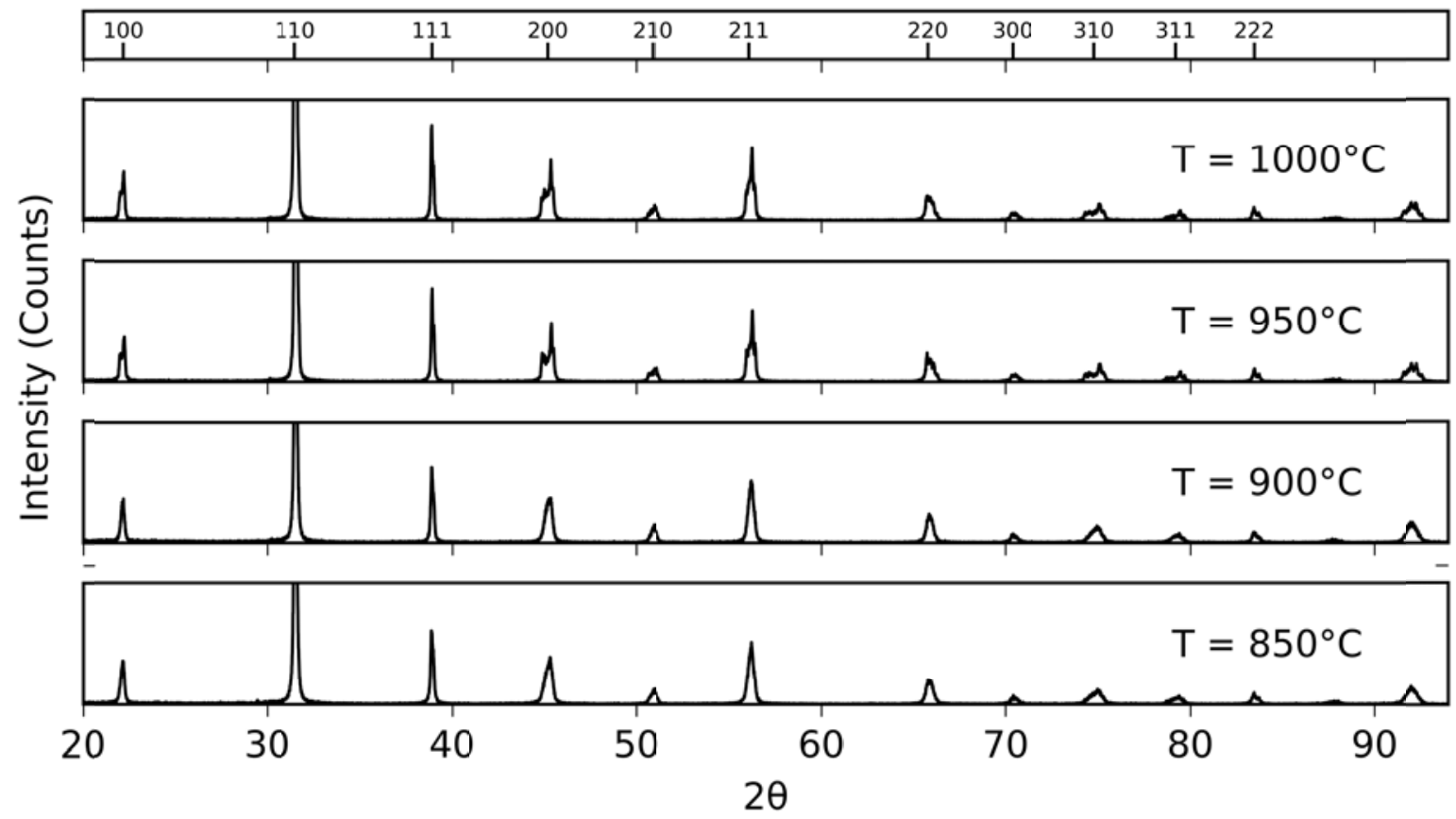


Figure 2

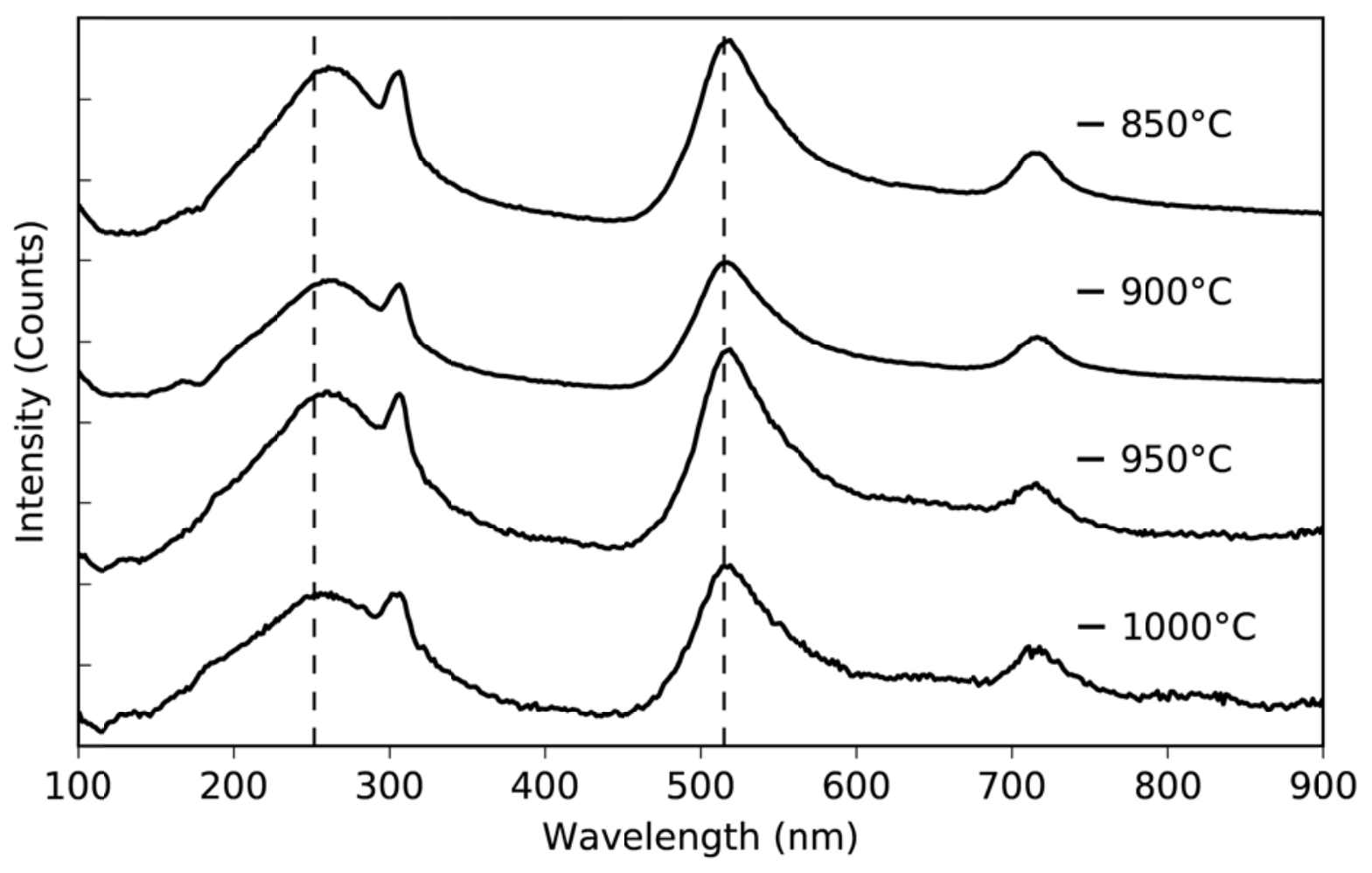


Figure 3
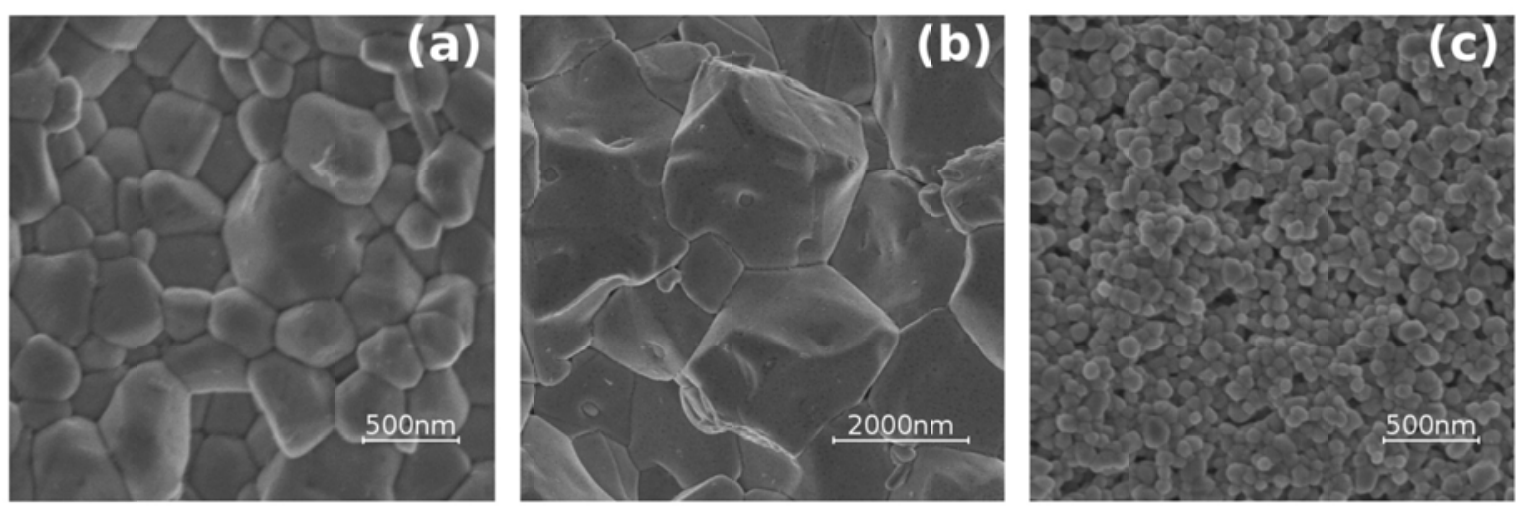
Figure 4

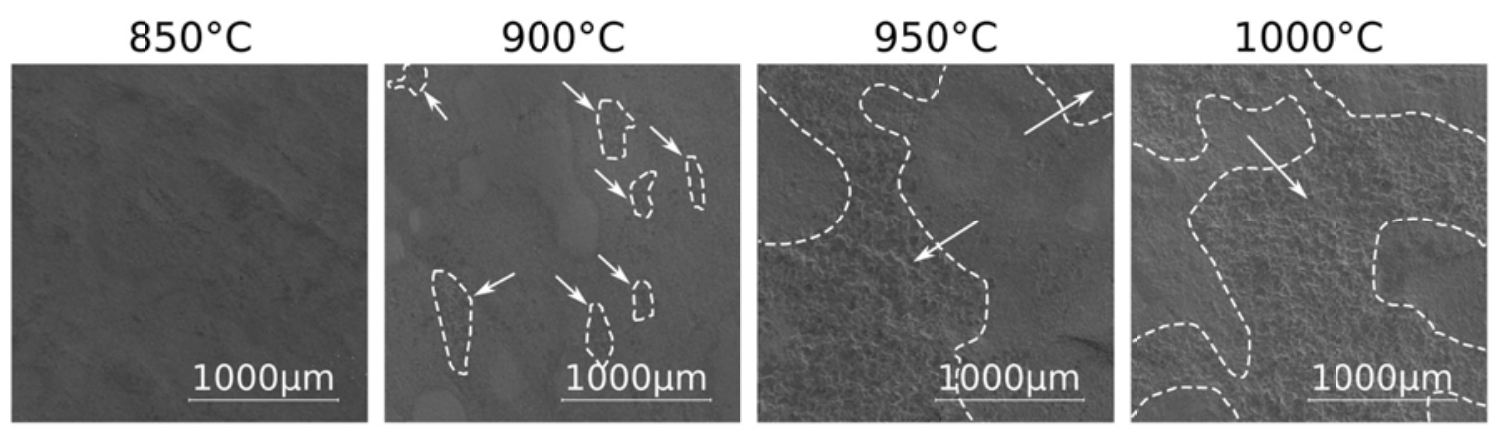


Figure 5
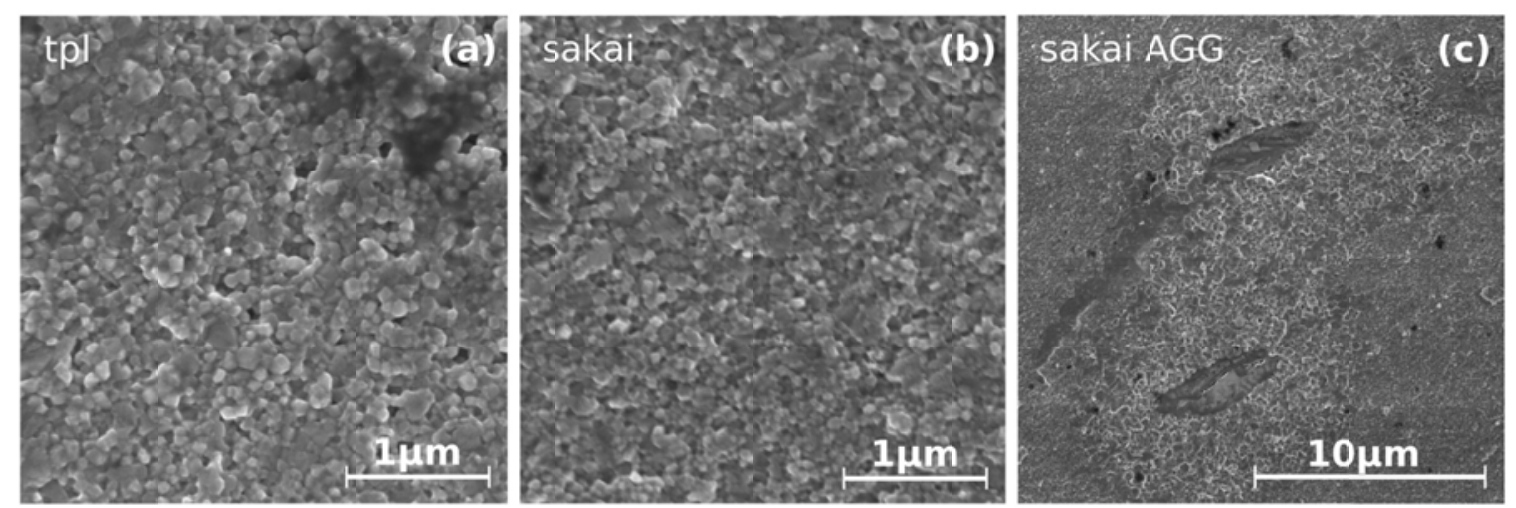
Figure 6
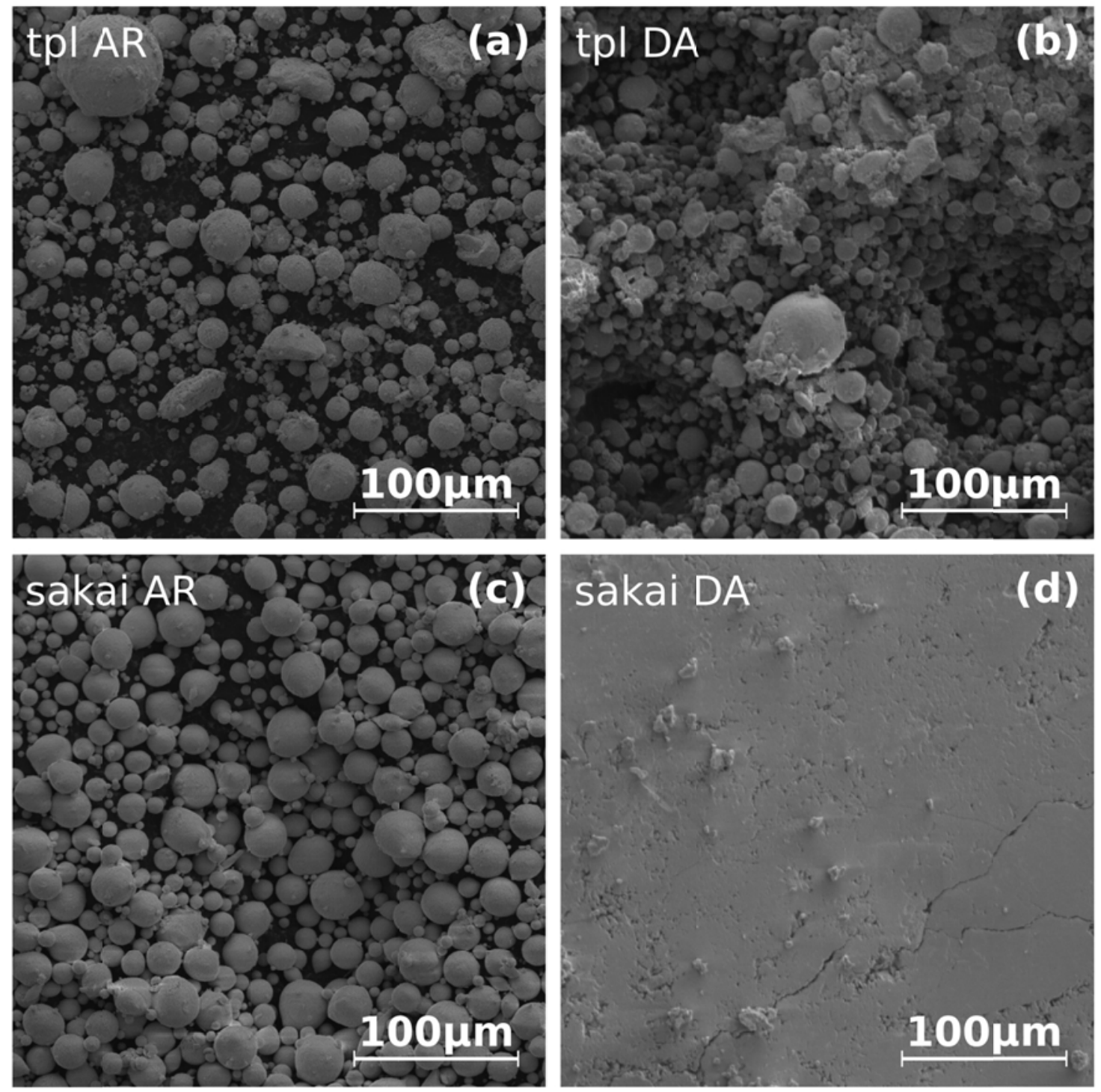
Figure 7

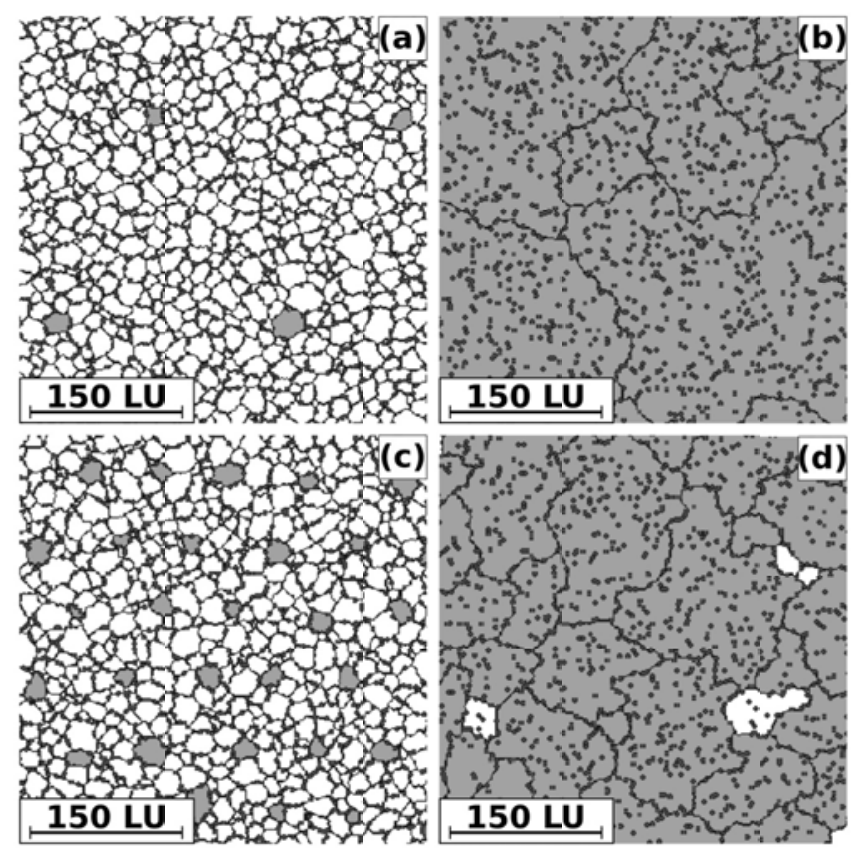

